\title{
BMG kündigt Reform der Approbationsordnung an
}

Zahlreiche Vertreter aus Politik, Zahnärzteschaft, Selbstverwaltung, Medien und Gesundheitswirtschaft haben am Frühjahrsfest von Kassenzahnärztlicher Bundesvereinigung (KZBV) und Bundeszahnärztekammer (BZ̈̈K) in der Britischen Botschaft in Berlin teilgenommen.

In seiner Begrüßung betonte der Vorstandsvorsitzende der KZBV, Dr. Wolfgang Eßer, die Anstrengungen der Zahnärzteschaft zur verbesserten zahnmedizinischen Versorgung von Pflegebedürftigen. „In den letzten zwei Jahren wurden über 2.700 Kooperationsverträge zwischen Zahnärzten und Pflegeeinrichtungen geschlossen. Wir freuen uns über diese positive Entwicklung“, sagte Eßer.

Der Staatssekretär im Bundesministerium für Gesundheit (BMG), Lutz Stroppe, kündigte in seiner Rede noch für dieses Jahr eine Reform der Approbationsordnung für Zahnärzte an. BZÄK-Präsident Dr. Peter Engel begrüßte die Neufassung der

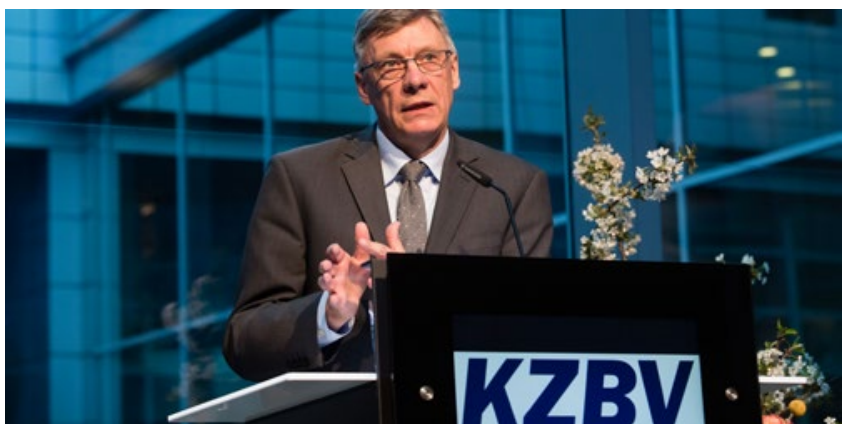

Gute Nachrichten von Lutz Stroppe, Staatssekretär im BMG. seit sechs Jahrzehnten nahezu unveränderten Approbationsordnung. „Damit können die Kolleginnen und Kollegen endlich nach einer Approbationsordnung praktizieren, die der modernen Zahnmedizin gerecht wird.“

Auch der Bundesverband der Zahnmedizinstudenten in Deutschland (BdZM) hat auf die Ankündigung aus dem BMG positiv reagiert. Dem vorausgegangen war die Ankündigung eines bundesweiten dreitägigen Protests der 13.000 Zahnmedizinstudenten für Mitte Mai, falls das BMG nicht seine jahrelange Verzögerungstaktik aufgäbe. „Der bundesweite Protest ist damit nicht endgültig vom Tisch“, sagte der Verbandsvorsitzende Kai Becker, „wir messen jetzt das BMG an seinem Versprechen, die Approbationsordnung tatsächlich umzusetzen und nicht, wie so oft, auf die lange Bank zu schieben." Die Erwartung der Studenten sei jetzt maximal hoch, und eine Verzögerung des Projekts werde einen Protest nach sich ziehen.

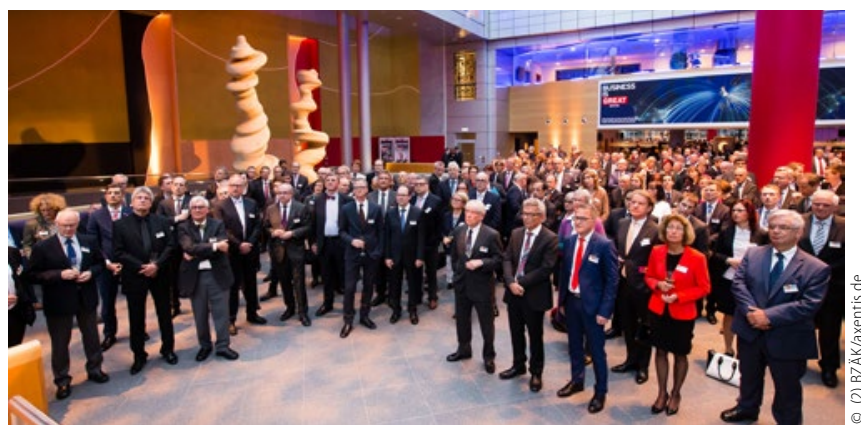

Die Branche trifft sich in der Britischen Botschaft.

\section{Barmer GEK Zahnreport}

\section{Kasse fordert mehr Transparenz}

Die zahnmedizinische Versorgung in Deutschland ist von Region zu Region unterschiedlich. Vor allem die Zuzahlungen für Zahnersatz variieren zwischen den Bundesländern. Das ist ein zentrales Ergebnis des im Mai veröffentlichten Zahnreports der Barmer GEK.

Wie der Report zeigt, zahlten die Patienten in Bayern 2014 durchschnittlich am meisten für Zahnersatz zu (1.132 Euro). Dahinter folgen Baden-Württemberg (1.097 Euro) und Hessen (993 Euro). Im Vergleich dazu lag der Eigenanteil für Zahnersatz in Sachsen durchschnittlich bei 637 Euro und in SachsenAnhalt lediglich bei 628 Euro. Aus Sicht des Barmer GEK-Vorstandsvorsitzenden, Christoph Straub, könne die unzureichende Aufklärung der Patienten über Vor- und Nachteile verschiedener Zahnersatzvarianten eine Erklärung für die Unterschiede sein: „Offensichtlich entscheiden sich immer mehr Menschen für Aspekte wie Komfort und Ästhetik, während Funktionalität und Haltbarkeit ins Hintertreffen geraten. Ist der Wunsch des Patienten wirklich immer Auslöser für diese Wahl?" Von der Wahl der Versorgung hänge auch die zahnärztliche Vergü- tung ab. Als Beispiel hierfür nannte Straub Zahnkronen. Laut Report wurden 2014 in Bayern weniger als zehn Prozent dieses Zahnersatzes in der Regelversorgung angefertigt. Mit Blick auf das Ergebnis forderte der Kassenchef mehr Transparenz in der zahnmedizinischen Versorgung: „So könnte man Vermutungen einer zu einseitigen Aufklärung der Patienten aus der Welt schaffen."

Der Präsident der Bundeszahnärztekammer (BZÄK), Dr. Peter Engel, wies den Vorwurf der mangelnden Aufklärung zurück: „Der Zahnarzt ist verpflichtet, über alle Alternativen der Therapie aufzuklären. Die Entscheidung für die Therapie ist abhängig von der Erwartungshaltung des Patienten und wird gemeinschaftlich mit dem Zahnarzt getroffen. Eine steigende Zahl an Patienten entscheidet sich dabei für eine hochwertige Versorgung mit einem Mehr an Lebensqualität und Ästhetik.“

Datengrundlage des aktuellen Barmer GEK Zahnreports sind die Abrechnungsdaten von 8,4 Mio. Versicherten im Jahr 2014. 